\title{
von Neumann Quantization of Aharonov-Bohm Operator with $\delta$ Interaction: Scattering Theory, Spectral and Resonance Properties
}

Gilbert HONNOUVO, Mahouton Norbert HOUNKONNOU and Gabriel Yves Hugues AVOSSEVOU

International Chair in Mathematical Physics and Applications (ICMPA)

07 BP 0196 Cotonou, Benin

and

Unité de Recherche en Physique Théorique (URPT), Institut de Mathématiques et de

Sciences Physiques (IMSP), 01 B.P. 2628 Porto-Novo, Bénin

E-mail:g_honnouvo@yahoo.fr; hounkonnou@yahoo.fr and avossevou@yahoo.fr

This article is part of the Proceedings titled "Geometrical Mathods in Physics: Bialowieza XXI and XXII"

\begin{abstract}
Using the theory of self-adjoint extensions, we study the interaction model formally given by the Hamiltonian $H_{\alpha}+V(r)$, where $H_{\alpha}$ is the Aharonov-Bohm Hamiltonian and $V(r)$ is the $\delta$-type interaction potential on the cylinder of radius $R$. We give the mathematical definition of the model, the self-adjointness of the Hamiltonian and provide relevant spectral properties, results for resonance effects and stationary scattering characteristics.
\end{abstract}

\section{Introduction}

The Aharonov-Bohm effect has received much attention in recent years $[2,5,6,8]$. Recently, Dabrowski and Stovicek described a quantum particle interacting with a thin solenoid and a magnetic flux with point interaction [6]. In this article, using the von Neumann theory of self-adjoint (s.a.) extensions of linear symmetric operators $[4,3,7,9]$ we investigate such physical properties as the stationary scattering theory, the spectral and resonance properties for the non relativistic Aharonov-Bohm type Hamiltonian formally expressed in polar coordinates as

$$
H_{\alpha}+V(r),
$$

where

$$
H_{\alpha}=-\frac{\partial^{2}}{\partial r^{2}}-\frac{1}{r} \frac{\partial}{\partial r}+\frac{1}{r^{2}}\left(i \frac{\partial}{\partial \phi}-\alpha\right)^{2}
$$

is the well known Aharonov-Bohm Hamiltonian acting in the Hilbert space $\mathcal{H}$;

$$
V(r)=\xi \delta(r-R), \quad \text { with } \xi \in \mathbb{R}, R>0 .
$$


In (1.2), we have fixed $\hbar=1, \quad m=1 / 2$. Besides, without loss of generality, we restrict our study to the case $0<\alpha<1$.

\section{The Model: Definition and Relevant Physical Properties}

Consider the radial equation for $\delta$ - cylinder interaction deduced from (1.1) using (1.2) and (1.3), and formally given by the expression:

$$
\left[-\frac{d^{2}}{d r^{2}}+\frac{(\alpha+m)^{2}-1 / 4}{r^{2}}+\xi_{m} \delta(r-R)\right] f_{m}(k, r)=k^{2} f_{m}(k, r) .
$$

Then, we assume the function $f_{m}(k, r)$ continuous at $r=R$ as follows:

$$
f_{m}\left(k, R_{+}\right)=f_{m}\left(k, R_{-}\right) \equiv f_{m}(k, R) .
$$

Integrating the equation (2.1) between $r=R-\epsilon$ and $r=R+\epsilon$ and taking the limit when $\epsilon \longrightarrow 0$, we have:

$$
f_{m}^{\prime}\left(k, R_{+}\right)-f_{m}^{\prime}\left(k, R_{-}\right)=\xi_{m} f(k, R) .
$$

Let us consider in $L^{2}\left(\mathbb{R}^{2}\right)$ the closed and non-negative operator $\dot{H}_{\alpha}=\overline{\left.H_{\alpha}\right|_{\left\{C_{0}^{\infty}\left(\mathbb{R}^{2} \backslash\{\partial \overline{\Gamma(O, R)}\}\right)\right\}}}$, with the domain

$$
D\left(\dot{H}_{\alpha}\right)=\left\{f \in L^{2}\left(\mathbb{R}^{2}\right) \cap H_{l o c}^{2,2}\left(\mathbb{R}^{2}\right) / f(\partial \overline{\Gamma(O, R)})=0, H_{\alpha} f \in L^{2}\left(\mathbb{R}^{2}\right)\right\},
$$

where $H_{l o c}^{m, n}(\Omega)$ is the local Sobolev space of indices $(\mathrm{m}, \mathrm{n})$. Let us now decompose the Hilbert space $\mathcal{H}=L^{2}\left(\mathbb{R}^{2}\right), L^{2}\left(\mathbb{R}^{2}\right)=L^{2}\left(\mathbb{R}^{+}\right) \otimes L^{2}\left(S^{1}\right), S^{1}$ being the unit circle in $\mathbb{R}^{2}$. The isomorphism $U$ is then introduced in order to remove the weight factor $r$ from the measure:

$$
U:\left\{\begin{array}{c}
L^{2}((0, \infty) ; r d r) \longrightarrow L^{2}((0, \infty) ; d r) \equiv L^{2}((0, \infty)) \\
f \longmapsto(U f)(r)=\sqrt{r} f(r),
\end{array}\right.
$$

so that we get the following decomposition of $L^{2}\left(\mathbb{R}^{2}\right)$ :

$$
L^{2}\left(\mathbb{R}^{2}\right)=\bigoplus_{m=-\infty}^{m=+\infty} U^{-1}\left(L^{2}\left(\mathbb{R}^{+}\right)\right) \bigotimes\left[\frac{e^{i m \phi}}{\sqrt{2 \pi}}\right], m \in \mathbb{Z}
$$

Provided this decomposition $\dot{H}_{\alpha}=\bigoplus_{m=-\infty}^{m=+\infty} U^{-1} \dot{h}_{\alpha, m} U \otimes \mathbb{1}$, where the operator $\dot{h}_{\alpha, m}$ in $L^{2}(] 0, \infty[)$ is defined by

$$
\dot{h}_{\alpha, m}=-\frac{d^{2}}{d r^{2}}+\frac{(\alpha+m)^{2}-1 / 4}{r^{2}}
$$

with the domain

$$
\begin{aligned}
\mathcal{D}\left(\dot{h}_{\alpha, m}\right)= & \left\{f \in L^{2}(] 0, \infty[, d r) \cap H_{l o c}^{2,2}(] 0, \infty[) ;\right. \\
& f\left(0_{+}\right)=0 \text { if }(\alpha+m)^{2}-1 / 4=0 ; f\left(R_{ \pm}\right)=0 ; \\
& \left.-f^{\prime \prime}+\left((\alpha+m)^{2}-\frac{1}{4}\right) r^{-2} f \in L^{2}((0, \infty))\right\}, m \in Z .
\end{aligned}
$$


The adjoint operator $\dot{h}_{\alpha, m}^{*}$ of $\dot{h}_{\alpha, m}$ is defined by

$$
\dot{h}_{\alpha, m}^{*}=-\frac{d^{2}}{d r^{2}}+\frac{(\alpha+m)^{2}-1 / 4}{r^{2}}
$$

with the domain

$$
\begin{aligned}
D\left(\dot{h}_{\alpha, m}^{*}\right)= & \left\{f \in L^{2}(] 0, \infty[, d r) \cap H_{l o c}^{2,2}(] 0, \infty[-\{R\}) ; f\left(0_{+}\right)=0\right. \text { if } \\
& (\alpha+m)^{2}-1 / 4=0 ; f\left(R_{+}\right)=f\left(R_{-}\right) \equiv f(R) ; \\
& \left.\left(-\frac{d^{2}}{d r^{2}}+\frac{(\alpha+m)^{2}-1 / 4}{r^{2}}\right) f \in L^{2}(] 0, \infty[)\right\}, m \in \mathbb{Z} .
\end{aligned}
$$

Consequently, we obtain $\dot{H}_{\alpha}^{*}=\bigoplus_{m=-\infty}^{m=+\infty} U^{-1} \dot{h}_{\alpha, m}^{*} U \otimes \mathbb{1}$. The indicial equation reads $h_{\alpha, m}^{*} f_{m}(k, r)=k^{2} f_{m}(k, r)$, or equivalently

$$
\left[-\frac{d^{2}}{d r^{2}}+\frac{(\alpha+m)^{2}-1 / 4}{r^{2}}\right] f_{m}(k, r)=k^{2} f_{m}(k, r) .
$$

Next, selecting, in the two-dimensional space of solutions, the solution which vanishes at the point $r=0$ and satisfies the boundary conditions (2.2) at $r=R$, we arrive at the function

$$
f_{|\alpha+m|}(k, r)=\left\{\begin{array}{l}
G_{|\alpha+m|-\frac{1}{2}}^{(0)}(k, R) \times F_{|\alpha+m|-\frac{1}{2}}^{(0)}(k, r) ; \quad r \leq R, \\
F_{|\alpha+m|-\frac{1}{2}}^{(0)}(k, R) \times G_{|\alpha+m|-\frac{1}{2}}^{(0)}(k, r) ; \quad r \geq R,
\end{array}\right.
$$

where

$$
\begin{aligned}
& F_{\nu}^{(0)}(k, r)=\left(\frac{k}{2}\right)^{-\nu-\frac{1}{2}} \Gamma\left(\nu+\frac{3}{2}\right) r^{\frac{1}{2}} J_{\nu+\frac{1}{2}}(k r), \\
& G_{\nu}^{(0)}(k, r)=\frac{-i \pi}{2} \frac{1}{\Gamma\left(\nu+\frac{3}{2}\right)}\left(\frac{k}{2}\right)^{\nu+\frac{1}{2}} r^{\frac{1}{2}} H_{\nu+\frac{1}{2}}^{(2)}(k r) .
\end{aligned}
$$

$J_{l}(z)$ and $H_{l}^{(2)}(z)$ are the Bessel and Hankel functions of order $l$, respectively [1]. Putting (2.12) into (2.11), we get

$$
f_{|\alpha+m|}(k, r)= \begin{cases}\frac{i \pi}{2} R^{1 / 2} H_{|\alpha+m|}^{(2)}(k R) r^{1 / 2} J_{|\alpha+m|}(k r) ; & r \leq R, \\ \frac{i \pi}{2} R^{1 / 2} J_{|\alpha+m|}(k R) r^{1 / 2} H_{|\alpha+m|}^{(2)}(k r) ; & r \geq R .\end{cases}
$$

Since the indicial equation admits one solution, $\dot{h}_{\alpha, m}$ has deficiency indices $(1,1)$ and, consequently, all self-adjoint (s.a) extensions of $\dot{h}_{\alpha, m}$ are given by a 1-parameter family of (s.a.) operators [4] which is defined by

$$
h_{\alpha, m, \xi_{m}}=-\frac{d^{2}}{d r^{2}}+\frac{(\alpha+m)^{2}-1 / 4}{r^{2}},
$$

with the domain

$$
\begin{aligned}
D\left(h_{\alpha, m, \xi_{m}}\right)= & \left\{f \in L^{2}(] 0, \infty[, d r) \cap H_{l o c}^{2,2}(] 0, \infty[\backslash\{R\}) ; f\left(0_{+}\right)=0\right. \text { if } \\
& (\alpha+m)^{2}-1 / 4=0 ; f\left(R_{+}\right)=f\left(R_{-}\right) \equiv f(R) ; f^{\prime}\left(R_{+}\right)-f^{\prime}\left(R_{-}\right)=\xi_{m} f(R) ; \\
& \left.\left(-\frac{d^{2}}{d r^{2}}+\frac{(\alpha+m)^{2}-1 / 4}{r^{2}}\right) f \in L^{2}(] 0, \infty[)\right\},
\end{aligned}
$$


$m \in \mathbb{Z},-\infty<\xi_{m} \leq+\infty$. The case $\xi_{m}=0$ coincides with the free kinetic energy Hamiltonian $\dot{h}_{\alpha, m, 0}$ for fixed quantum number $m$. Let $\xi=\left\{\xi_{m}\right\}_{m \in \mathbb{Z}}$ and introduce in $L^{2}\left(\mathbb{R}^{2}\right)$ the operator

$$
H_{\alpha, \xi}=\bigoplus_{m=-\infty}^{m=+\infty} U^{-1} h_{\alpha, m, \xi_{m}} U \bigotimes \mathbb{1}
$$

By definition, $H_{\alpha, \xi}$ is the rigorous mathematical formulation of the formal expression (1.1). Actually, it provides a slight generalization of (1.1), since $\xi$ may depend on $m \in \mathbb{Z}$.

\subsection{The resolvent equation}

We get the following:

Theorem 1. (i) The resolvent of $h_{\alpha, m, \xi_{m}}$ is given by

$$
\left(h_{\alpha, m, \xi_{m}}-k^{2}\right)^{-1}=\left(h_{\alpha, m, 0}-k^{2}\right)^{-1}+\mu_{m}(k)\left(f_{|\alpha+m|}(-\bar{k}), .\right) f_{|\alpha+m|}(k),
$$

$k^{2} \in \rho\left(\dot{h}_{\alpha, m, \xi_{m}}\right), \mathcal{I} m(k)>0 ; m \in \mathbb{Z}$, where $\mu_{m}(k)=-\xi_{m}\left[1+\xi_{m} g_{m, k}(R, R)\right]^{-1}$ and $\left(h_{\alpha, m, 0}-k^{2}\right)^{-1}$, is the free resolvent with integral kernel

$$
g_{m, k}\left(r, r^{\prime}\right)= \begin{cases}G_{|\alpha+m|-\frac{1}{2}}^{(0)}(k, r) \times F_{|\alpha+m|-\frac{1}{2}}^{(0)}\left(k, r^{\prime}\right) ; & r^{\prime} \leq r, \\ F_{|\alpha+m|-\frac{1}{2}}^{(0)}(k, r) \times G_{|\alpha+m|-\frac{1}{2}}^{(0)}\left(k, r^{\prime}\right) ; \quad r^{\prime} \geq r .\end{cases}
$$

We note that $g_{m, k}(R, r)=f_{|\alpha+m|}(k, r), \mathcal{I} m(k)>0$.

(ii) The resolvent of $H_{\alpha, \xi}$ is given by

$$
\left(H_{\alpha, \xi}-k^{2}\right)^{-1}=\left(H_{\alpha, 0}-k^{2}\right)^{-1}+\bigoplus_{m=-\infty}^{m=+\infty} \mu_{m}(k)\left(|.|^{-1} f_{|\alpha+m|}(-\bar{k}) \frac{e^{i m \phi}}{\sqrt{2 \pi}}, .\right)|\cdot|^{-1} f_{|\alpha+m|}(k) \frac{e^{i m \phi}}{\sqrt{2 \pi}},
$$

$k^{2} \in \rho\left(H_{\alpha, \xi}\right), \mathcal{I} m(k)>0$.

Theorem 2. The domain $D\left(h_{\alpha, m, \xi_{m}}\right)$ consists of functions of the type $\psi_{m}(k, r)=F_{\alpha, m}(k, r)+$ $\mu_{m}(k) F_{\alpha, m}(k, R) g_{m, k}(R, r), F_{\alpha, m} \in D\left(h_{\alpha, m, 0}\right)$ and $k^{2} \in \rho\left(h_{\alpha, m, \xi_{m}}\right), \mathcal{I} m(k)>0$. This decomposition is unique and with $\psi_{m} \in D\left(h_{\alpha, m, \xi_{m}}\right)$ of this form, we obtain $\left(h_{\alpha, m, \xi_{m}}-k^{2}\right) \psi_{m}=$ $\left(h_{\alpha, m, 0}-k^{2}\right) F_{\alpha, m}$.

Proof. One may follow step by step [3], where a similar result was obtained for point interaction.

\subsection{Spectral properties}

Spectral properties of $h_{\alpha, m, \xi_{m}}$ are provided by the following theorem where $\sigma(),. \sigma_{e s s}($.$) ,$ $\sigma_{a c}(),. \sigma_{s c}($.$) and \sigma_{p}($.$) denote the spectrum, essential spectrum, absolutely continuous$ spectrum, singularly continuous spectrum and point spectrum, respectively.

Theorem 3. For all $\xi_{m} \in(-\infty, \infty), \sigma_{e s s}\left(h_{\alpha, m, \xi_{m}}\right)=\sigma_{a c}\left(h_{\alpha, m, \xi_{m}}\right)=[0, \infty), \sigma_{s c}\left(h_{\alpha, m, \xi_{m}}\right)=$ $\emptyset, \sigma_{p}\left(h_{\alpha, m, \xi_{m}}\right) \cap[0, \infty)=\emptyset$. The negative eigenvalues of $h_{\alpha, m, \xi_{m}}$ are obtained from the equation $1+\xi_{m} g_{m, i \sqrt{-E}}(R, R)=0, E<0$, which has at most one solution $E_{0}<0$. 


\subsection{Resonances of $h_{\alpha, m, \xi_{m}}$}

Using the boundary conditions, the resolvent equation is given by

$$
\left(h_{\alpha, m, \xi_{m}}-k^{2}\right)^{-1}=\left(h_{\alpha, m, 0}-k^{2}\right)^{-1}-\xi_{m}\left[1+\xi_{m} g_{m, k}(R, R)\right]^{-1}\left(f_{\alpha, m}(-\bar{k}), .\right) f_{\alpha, m}(k)
$$

$k^{2} \in \rho\left(h_{\alpha, m, \xi_{m}}\right), \mathcal{I} m(k)>0 ; m \in \mathbb{Z}$. The resonance equation is then $1+\xi_{m} g_{m, k}(R, R)=0$, or equivalently $1-\xi_{m} i \frac{\pi}{2} R H_{|\alpha+m|}^{(2)}(k R) J_{|\alpha+m|}(k R)=0$. This equation generates an infinite set of resonances off the imaginary axis for $h_{\alpha, m, \xi_{m}}$, whatever the partial wave characterized by the quantum number $m$ and $\xi_{m}$ [8]. The only difficulties one encounters to prove this statement arise from the complexity of the mathematical expressions that appear more and more less treatable analytically as the quantum number $m$ raises. For instance, taking just $\alpha=1 / 2$ and $m=0$ or -1 leads to an intricate nonlinear system. Numerical computations allow to go round these difficulties.

\subsection{Stationary Scattering Theory for the pair $\left(h_{\alpha, m, \xi_{m}} ; h_{\alpha, m, o}\right)$}

The phase shifts of $h_{\alpha, m, \xi_{m}}$ may be obtained through the asymptotic behavior of $\mathcal{F}_{m, \alpha, \xi_{m}}(k, r)$ as $r \longrightarrow \infty$. So doing, we get

$$
\begin{array}{rll}
\mathcal{F}_{m, \alpha, \xi_{m}}(k, r) \quad \stackrel{k>0}{\longrightarrow} & A_{m}(k) \sin \left(k r-\frac{\pi(|\alpha+m|-1 / 2)}{2}\right)+ \\
& +\mu_{m}(k) F_{|\alpha+m|-1 / 2}^{(0)}(k, R) F_{|\alpha+m|-1 / 2}^{(0)}(k, r) B_{m}(k) \\
& \times \exp \left[-i\left(k r-\frac{\pi(|\alpha+m|-1 / 2)}{2}\right)\right] \\
= & {\left[C_{1, m}^{2}(k)+C_{2, m}^{2}(k)\right]^{1 / 2} \sin \left(k r-\frac{\pi(|\alpha+m|-1 / 2)}{2}+\delta_{m, \xi_{m}}(k)\right)} \\
& +o(1)
\end{array}
$$

and the phase shifts express as

$$
\delta_{m, \xi_{m}}(k)=-\arctan \frac{B_{m}(k) \mu_{m}(k)\left(F_{|\alpha+m|-1 / 2}^{(0)}(k, R)\right)^{2}}{A_{m}(k)-i B_{m}(k) \mu_{m}(k)\left(F_{|\alpha+m|-1 / 2}^{(0)}(k, R)\right)^{2}}
$$

where $A_{m}(k)=2^{-(|\alpha+m|-1 / 2)}\left(k^{-|\alpha+m|-1 / 2}\right) \Gamma(2|\alpha+m|+1) \Gamma(|\alpha+m|+1 / 2)^{-1}$ and $B_{m}(k)=$ $1 /\left(k A_{m}(k)\right)$. The corresponding on-shell scattering matrix is defined by

$$
S_{m, \xi_{m}}(k)=1-2 i k B_{m}^{2}(k) \mu_{m}(k)\left(F_{|\alpha+m|-1 / 2}^{(0)}(k, R)\right)^{2},
$$

while the on-shell scattering amplitude $f_{\xi}\left(k, \omega, \omega^{\prime}\right)$ corresponding to $H_{\alpha, \xi}$ is given by

$$
f_{\xi}\left(k, \omega, \omega^{\prime}\right)=4 \pi \sum_{m=-\infty}^{m=+\infty} f_{m, \xi_{m}}(k)\left[\frac{e^{-i m \omega^{\prime}}}{\sqrt{2 \pi}}\right]\left[\frac{e^{i m \omega}}{\sqrt{2 \pi}}\right]
$$


$k \geq 0 \quad ; \quad \omega, \omega^{\prime} \in S^{1}$. The partial wave scattering amplitude $f_{m, \xi_{m}}(k)$ reads

$$
f_{m, \xi_{m}}(k)=-B_{m}^{2}(k) \mu_{m}(k)\left(F_{|\alpha+m|-1 / 2}^{(0)}(k, R)\right)^{2} .
$$

The on-shell scattering operator $S_{\xi}(k)$ in $L^{2}\left(S^{1}\right)$ corresponding to $H_{\alpha, \xi}$ is defined by

$$
\left(S_{\xi}(k) \phi\right)(\omega)=\phi(\omega)-\frac{k}{2 \pi i} \int_{S^{1}} d \omega^{\prime} f_{\xi}\left(k, \omega, \omega^{\prime}\right) \phi\left(\omega^{\prime}\right),
$$

$k \geq 0 \quad ; \quad \omega, \omega^{\prime} \in S^{1}, S_{\xi}(k)=1+2 i k \sum_{m=-\infty}^{m=+\infty} f_{m, \xi_{m}}(k)\left(\frac{e^{i m(\cdot)}}{\sqrt{2 \pi}},.\right) \frac{e^{i m(w)}}{\sqrt{2 \pi}}$.

Acknowledgments. M N H thanks Professor S. T Ali (Canada) and Professor A. Odzijewicz (Poland) for the hospitality and invitation at XXI Workshop on Geometric Methods in Physics (XXI WGMP), Recent Developments in Quantization.

\section{References}

[1] Abramowitz M and Stegun I A, Handbook of Mathematical Functions, Dover, New York, 1972.

[2] Aharonov Y and Bohm D, Significance of Electromagnetic Potentials in the Quantum Theory, Phys. Rev. 115, (1959), 485-491.

[3] Albeverio S, Gesztesy F, Hoegh-Krohn R and Holden H, Solvable Models in Quantum Mechanics, Texts and Monographs in Physics, Springer Verlag, Berlin, 1988.

[4] Akhiezer N I and Glazman I M, Theory of Linear Operators in Hilbert Space, Vol. 2, Pitman, Boston, 1981.

[5] Ballentine L E, Quantum Mechanics, World Scientific, Singapore, New Jersey, London, HongKong, 1995.

[6] Dabrowski L and Stovicek P, Aharonov - Bohm Effect with $\delta$ - Type Interaction, J. Math. Phys. 39, (1998), 47 - 62.

[7] Hounkonnou M N, Hounkpe M and Shabani J, Scattering Theory for Finitely Many Sphere Interactions Supported by Concentric Spheres, J. Math. Phys. 38, (1997), 2832 - 2850.

[8] Honnouvo G, Hounkonnou M N and Avossevou G H Y, Aharonov-Bohm Type Operator with $\delta$ and $\delta^{\prime}$ Interactions: Scattering Theory, Spectral and Resonance Properties, to publish.

[9] Reed M and Simon B, Methods of Modern Mathematical Physics, 4, Analysis of Operators, Academic, New York, 1978. 